\title{
Structural comparison between natural teeth and implants
}

\author{
Ye Jin LEE, So Hyeon LEE, Kyung Sook HWANG* \\ Dept. of Dent. Tech., Shin Han Univ., Korea
}

\begin{abstract}
The main discourse: A dental implant is a prosthesis that utilizes an artificial tooth root implanted in the bone instead of a lost tooth, and is usually combined with three components that are fastened by screws. The implant itself is a part of the tooth structure that corresponds to the root of the tooth and is called a fixture, which is embedded in the alveolar bone to be combined. In addition, it is a part of the oral cavity that corresponds to the head of a natural tooth, called a crown, and actually participates in mastication. A device that connects the implant fixture to the location where the tooth is lost is implanted and the crown prosthesis is put on after the bone is combined with the gum bone and is called an abutment. In this way, the fixture, abutment, and crown prosthesis are collectively referred to as an implant. These three types of structures are commonly connected to each other with screws or cement. This is a treatment method that requires at least 2-3 months of osseointegration as the fixture must be directly attached to the bone after being buried in the alveolar bone. However, although natural teeth and implants are similar in appearance, there is a big difference in histology and anatomy. That is, there are no nerves and blood vessels inside the fixture like natural teeth, and there is no periodontal ligament.

In general, bending force is generated in the implant by the occlusal force of the oral cavity, which causes mechanical fracture of the implant or screw loosening. Through many studies, loosening of the screw appears to be the main clinical problem in single-tooth implants, unlike many implant prostheses, and loosening of the prosthesis due to loosening of the screw is a problem in the implant fixture and retaining screw. is highlighted After all, the structure of these implants is a phenomenon that occurs without the patient's awareness because there are no nerves and blood vessels inside, making it impossible to avoid risk. In addition, the implant fixture is made of a metal called titanium, and surface treatment and surface structure form are very important so that it can be well combined with the gingival bone. However, as it is an osseointegration method that directly attaches to the teeth, the periodontal ligament of natural teeth cannot be shaped. In conclusion, natural teeth and implants recovered their masticatory power due to the similarity in appearance, but their defensive ability or avoidance response was defective due to the histological and anatomical differences inside the implant. This is the biggest difference.
\end{abstract}

Keywords: advantage of implant, natural teeth, histological, anatomical

Acknowledgement: This study is supported by Uniance's Central Research Institute.

Copyright (C) 2021. Korean Academy of Preventive Dentistry. All rights reserved.

This is an Open Access article distributed under the terms of the Creative Commons Attribution Non-Commercial License (http://creativecommons.org/licenses/ by-nc/4.0) which permits unrestricted non-commercial use, distribution, and reproduction in any medium, provided the original work is properly cited. 